\title{
En niños prescolares el tratamiento intermitente con budesonide fue igual de eficaz al tratamiento diario para el control del broncoespasmo recurrente
}

Daily or intermittent budesonide showed similar efficacy to control recurrent wheezing in preschool children

Zeiger RS y col. N Engl J Med. 2011;365(21):1990-2001.

\section{Objetivo}

Evaluar si el tratamiento crónico con budesonide inhalado a dosis bajas es superior a un esquema intermitente a dosis mayores, en niños con antecedentes de broncoespasmo y sospecha de asma.

\section{Diseño y lugar}

Estudio clínico aleatorizado*, controlado con grupo tratamiento paralelo*, doble ciego*, realizado en siete centros en EE.UU, con un seguimiento de 52 semanas.

\section{Pacientes}

Se incluyeron niños de 12 a 53 meses de edad con un índice predictivo de asma modificado (API, por sus siglas en inglés) positivo, que presentaran cuatro o más episodios de broncoespasmo en el último año y al menos una exacerbación que requiriese el uso de corticoides sistémicos, atención de urgencia y/u hospitalización. Se excluyeron aquellos pacientes que utilizaron corticoides sistémicos en mas de seis oportunidades y que requirieron internación más dos veces en el último año, respectivamente; y quienes además, durante las dos semanas iniciales del estudio, requirieron el uso de albuterol tres o más días por semana o presentaron dos o más despertares durante la noche debido a broncoespasmo.Se reclutaron 139 pacientes en cada rama. Completaron el estudio 100 en el grupo de budesonide inhalado diario, y 113 en el intermitente. Las características demográficas fueron similares en ambos grupos.

\section{Intervención}

Ante la presencia síntomas desencadenantes de un episodio de broncoespasmo, los padres debían iniciar en forma precoz un "kit de tratamiento", diferente según la rama de tratamiento (ver tabla 1).
Tabla 1: diseño de los grupos de intervención.

\begin{tabular}{|c|c|c|c|}
\hline $\begin{array}{l}\text { Fase inicial } \\
\text { (2 semanas) }\end{array}$ & \multicolumn{3}{|c|}{ Fase de tratamiento (52 semanas) } \\
\hline \multirow{4}{*}{$\begin{array}{l}\text { Placebo } 1 \\
\text { vez por } \\
\text { noche }+ \\
\text { albuterol a } \\
\text { demanda }\end{array}$} & Grupo de tratamient & Tipo de intervención diar & 0 de intervenció \\
\hline & aleatorizado & excepto durante CVAS & bor siete diz \\
\hline & Diario & Budesonide $(0,5 \mathrm{mg})$ & $\begin{array}{l}\text {-Placebo por la mañana } \\
\text {-Budesonide } \\
(0,5 \mathrm{mg}) \text { por la noche }\end{array}$ \\
\hline & Intermitente & Placebo & $\begin{array}{c}\text {-Budesonide } \\
\text { (1 mg) por la mañana } \\
\text {-Budesonide } \\
(1 \mathrm{mg}) \text { por la noche }\end{array}$ \\
\hline
\end{tabular}

CVAS: cuadro de vías aéreas.

\section{Medición de resultados}

Se evaluó como resultado primario* la frecuencia de episodios de broncoespasmo que requirieron corticoides sistémicos. Entre los resultados secundarios* se evaluó: tiempo hasta la exacerbación; tasa de fallo de tratamiento; tasa de utilización de servicios de salud; efecto en el crecimiento; número de días sin síntomas; severidad de los síntomas durante una exacerbación; uso de albuterol.

\section{Resultados}

No se econtraron diferencias significativas tanto para los resultados primarios como secundarios entre ambas estrategias de tratamiento.

Tabla 1: frecuencia de exacerbaciones asmáticas que requirieron corticoides sistémicos por rama de tratamiento.

\begin{tabular}{l|c|c|c}
\multirow{2}{*}{ Resultado } & $\begin{array}{c}\text { Tratamiento intermitente } \\
(\mathbf{n = 1 3 9 )}\end{array}$ & $\begin{array}{c}\text { Tratamiento diario } \\
(\mathbf{n = 1 3 9 )}\end{array}$ & $\begin{array}{c}\mathbf{R} \\
\mathbf{( I C 9 5 \% )}\end{array}$ \\
\cline { 2 - 3 } & \multicolumn{2}{|c|}{ Tasa de evento/persona-año (95\% IC) } & \\
\hline $\begin{array}{l}\text { Ciclos de } \\
\text { corticoides } \\
\text { sistémicos } \\
\text { por asma }\end{array}$ & $0,95(0,75$ a 1,20) & $0,97(0,76$ a 1,22) & $0,99(0,71$ a 1,35) \\
\hline
\end{tabular}

\section{Conclusiones del estudio}

El esquema de tratamiento con bajas dosis bajas de bude-sonide no demostró ser superior al esquema intermitente con dosis altas. El primer esquema conllevo una mayor exposición a budesonide al año de tratamiento.

Fuente de financiamiento: National Heart, Lung, and Blood Institute; Washington University School of Medicine.

\section{Comentario}

En general, los niños menores de cinco años con episodios recurrentes de broncoespasmo (mas de cuatro en el último año) y riesgo incrementado de asma (valorado por API ${ }^{\S}$ ) tienen indicación de iniciar tratamiento preventivo con corticoides inhalados en forma diaria ${ }^{1,2}$. Sin embargo, este tratamiento despierta dudas con respecto a los potenciales efectos adversos de los corticoides. Este estudio ha sido diseñado como un ensayo de superioridad $^{*}$ y no de bioequivalencia ${ }^{*, 4}$; la magnitud del intervalo de confianza de la tasa relativa de exacerbaciones no permiten afimar en forma taxativa que ambos tratamientos tengan eficacia similar, ya que uno u otro podrían resultar hasta un $35 \%$ mas efectivos.
El presente estudio podría dar el puntapié para considerar este esquema de tratamiento en niños pequeños que resulten similares a los aquí estudiados. Sin embargo, la elección del esquema intermitente deberá basarse en una menor exposición acumulada a glucocorticoides, más que en potenciales beneficios sobre el crecimiento (tampoco demostrados aqui). Por otro lado resulta importante mencionar que el esquema intermitente requiere ser instaurado ante la identificación precoz de síntomas específicos, lo cual implica un mayor entrenamiento y disciplina por parte de los padres.

María Victoria Salgado [ Servicio de Medicina Familiar y Comunitaria del hospital Italiano de Buenos Aires. maria.salgado@ hospitalitaliano.org.ar ]

Salgado MV. En niños prescolares el tratamiento intermitente con budesonide fue igual de eficaz al tratamiento diario para el control del broncoespasmo recurrente. Evid Act Práct Ambul. Jul-Set 2012;15(3):87. Comentado de: Ziger Rs y col. Daily or intermittent budesonide in preschool children with recu-rrent wheezing. N Engl J Med. 2011 Nov 24;365(21):1990-2001. PMID: 22111718.

\section{Referencias}

1. Guilbert TW, Lemanske RF. Wheezing phenotypes and prediction of asthma in young children. In: UpToDate, Basow, DS (Ed), UpToDate, Waltham, MA, 2012 2. Sawicki G, Haver K. Chronic asthma in children younger than 12 years: Controller medications. In: UpToDate, Basow, DS (Ed), UpToDate, Waltham, MA, 2012 3. Christensen E, "Methodology of superiority vs. equivalence trials and non-inferiority trials". J Hepatol. 2007 May;46(5):947-54.

4. Lesaffre E, "Superiority, Equivalence, and Non-Inferiority Trials". Bulletin of the NYU Hospital for Joint Diseases 2008;66(2):150-4.

${ }^{5} \mathrm{API}$ toma en cuenta el antecedente de asma en los padres y diagnóstico clínico de eccema en los niños como criterios mayores; y el diagnóstico de rinitis alérgica, sibilancias fuera de episodios de CVAS y eosinofilia $>4 \%$ como criterios menores. La presencia de un criterio mayor y dos criterios menores en un niño menor de cinco años con episodios recurrentes de sibilancias aumenta la probabilidad del diagnóstico futuro de asma entre cuatro y siete veces. El puntaje tiene baja sensibilidad ${ }^{*}$ pero un elevado valor predictivo negativo* lo cual resulta útil para identificar a los niños con baja probabilidad de asma futuro. 